\section{Estudio histomorfométrico del hueso cortical en rebordes edéntulos y su relación con la tomografía computarizada cone beam. Resultados preliminares}

\author{
Histomorphometric study of cortical bone \\ in edentulous ridges and its relationship \\ with cone beam computed tomography. \\ Preliminary results
}

\begin{abstract}
Resumen
Objetivo. Determinar la relación de la calidad ósea del hueso cortical según las diferentes regiones del maxilar y la mandíbula mediante la histomorfometría y los valores Hounsfield equivalentes de la tomografía computarizada cone beam (CBCT) en pacientes parcialmente edéntulos. Métodos. Este estudio transversal analizó la histomorfometría según número de osteonas, osteocitos y espesor del hueso cortical y las unidades Hounsfield en la $C B C T$ según sexo y regiones del maxilar y la mandíbula para determinar la correlación existente en 29 pacientes de la Clínica de Posgrado de la Facultad de Odontología de la Universidad Nacional Mayor de San Marcos. Resultados. El promedio de las unidades Hounsfield fue $593 \pm 121,10$ y $570 \pm 127,35$ en el sexo masculino y femenino respectivamente. El número de osteones en promedio fue de $16,5 \pm 2,71$ y $12 \pm 4,68$ en el sexo masculino y femenino respectivamente. El número de osteocitos promedio fue $179 \pm 39,99$ en el sexo masculino y $124 \pm 50,55$ en el femenino. El espesor cortical en el sexo masculino fue $33 \pm 50,55$ micras y $31 \pm 8,91$ en el femenino. Se realizó la correlación de Pearson entre las unidades Hounsfield y las variables espesor cortical, número de osteones, y número de osteocitos. Conclusión. Existe una correlación positiva moderada estadísticamente significativa entre el espesor del hueso cortical y las unidades Hounsfield $(\mathrm{r}=0,452, \mathrm{p}<0,01)$.
\end{abstract}

Palabras clave: Proceso alveolar; Tomografía computarizada por rayos X; Histología (fuente: DeCS BIREME).

\begin{abstract}
Objective. Determine the relationship of bone quality in cortical bone according to the different regions of the maxilla and mandible by histomorphometry and the equivalent Hounsfield values of Cone Beam computed tomography in partially edentulous patients. Methods. This cross-sectional study analyzed histomorphometry according to the number of osteons, osteocytes and thickness of the cortical bone and the Hounsfield units in the Cone Beam computed tomography according to gender and regions of the maxilla
\end{abstract}

Artículo Original

Sixto García Linares ${ }^{1, a}$, Luis Gálvez Calla 1,b

${ }^{1}$ Universidad Nacional Mayor de San Marcos, Facultad de Odontología. Lima, Perú.

${ }^{a}$ Magister en Estomatología.

${ }^{\mathrm{b}}$ Doctor en Odontología.

\section{Correspondencia:}

Sixto García Linares: sgarcia@@unmsm.edu.pe Universidad Nacional Mayor de San Marcos, Facultad de Odontología. Calle Germán Amézaga 375. Lima 1, Perú. ORCID: 0000-0001-5057-5900

\section{Coautores:}

Luis Gálvez Calla: Igalvezc@unmsm.edu.pe ORCID: 0000-0002-6112-4993

Editor:

Juan Carlos Cuevas-González

Universidad Autónoma de Ciudad Juárez, México.

Conflicto de intereses: no existe conflicto de intereses.

Fuente de financiamiento: trabajo financiado por el fondo de subvención de tesis de postgrado CODIGO A18050046

Recibido: 04/12/19

Aceptado: $16 / 04 / 20$

Publicado: 04/08/20

(C) Los autores. Este artículo es publicado por la revista Odontología Sanmarquina de la Facultad de Odontología, Universidad Nacional Mayor de San Marcos. Este es un artículo de acceso abierto, distribuido bajo los términos de la licencia Creative Commons Atribucion - No Comercia_Compartir Igual 4.0 Internacional. (http://creativecommons.org/licenses/by-nc-sa/4.0/) que permite el uso no comercial, distribución y reproducción en cualquier medio, siempre que la obra original sea debidamente citada. 
and mandible to determine the existing correlation in 29 patients from Graduate Clinic of The Faculty of Dentistry at the National University of San Marcos. Results. Hounsfield units average was $593 \pm 121.10$ in the male gender and $570 \pm 127.35$ in the female gender. number of osteons average was $16.5 \pm 2.71$ male and $12 \pm 4.68$ in the female. Number of osteocytes average was $179 \pm 39.99$ male and $124 \pm 50.55$ in the female. About cortical alveolar bone thickness in males, it was $33 \pm 50.55$ microns and $31 \pm$ 8.91 in females. It was found normal distribution between the data. Pearson's correlation between Hounsfield units and quantitative variables cortical thickness, number of osteons, and number of osteocytes. Conclusion. There is a statistically significant moderate positive correlation between the thickness of the cortical bone and the Hounsfield units $(\mathrm{r}=0.452, \mathrm{p}<0.01)$.

Keywords: Alveolar process; Tomography, X-ray computed; Histology (source: MeSH NLM).

\section{Introducción}

Una alternativa de tratamiento para la pérdida dentaria son los implantes dentales ${ }^{1,2}$. Un implante dental es una pieza de un biomaterial que se inserta en el hueso de la mandíbula o el maxilar para reemplazar la raíz de un diente ${ }^{1}$. La oseointegración es la conexión firme, estable y duradera entre el implante dental y el tejido óseo formado a su alrededor ${ }^{3}$. Esta formación de hueso está sujeta a los eventos de migración, proliferación y diferenciación celular que suceden en la denominada interfase hueso-implante dental ${ }^{4}$. Es importante conocer la calidad ósea para la planificación y pronóstico de la cirugía de implantes dentales puesto que la interfase hueso implante debe ser una relación estable y duradera en el tiempo ${ }^{1,5}$.

La calidad ósea considerada por algunos autores como densidad ósea ${ }^{6}$ se refiere a la calidad del espesor del hueso cortical en términos de resistencia a la estabilidad mecánica en relación al implante dental ${ }^{3,6}$.

Lekholm y Zarb propusieron una clasificación de la calidad del hueso cortical y medular que podría relacionarse con las diferentes zonas del maxilar y la mandíbu$\mathrm{la}^{3}$. Tipo 1: se compone casi exclusivamente de hueso compacto homogéneo. Éste era el ideal con presencia de algunas trabéculas espaciadas con pequeños espacios medulares. Tipo 2: el hueso compacto ancho rodea el esponjoso denso. Esta parte esponjosa presenta espacios medulares ligeramente mayores con menor uniformidad en el patrón óseo. Según los autores este hueso era suficiente para los implantes. Tipo 3: la cortical delgada rodea el hueso esponjoso denso. Grandes espacios medulares entre las trabéculas óseas. Este hueso según los autores provocaba la desadaptación del implante. Tipo 4: la cortical delgada rodea el abundante hueso esponjoso poco denso.

El objetivo de este trabajo es determinar la relación de la calidad ósea del hueso cortical según las diferentes regiones del maxilar y la mandíbula mediante la histomorfometría y los valores Hounsfield de la tomografía computarizada cone beam en pacientes parcialmente edéntulos mostrando los resultados preliminares.

\section{Métodos}

Este es un estudio observacional, descriptivo y transversal. Fueron incluidos todos los pacientes programados para cirugía de implantes dentales de la clínica de postgrado de periodoncia de la Facultad de Odontología de la Universidad Nacional Mayor de San Marcos en los años 2018 y 2019 que cumplían los criterios de inclusión y exclusión. Los instrumentos utilizados han sido ampliamente validados.

La selección de muestra fue no probabilística por conveniencia. Los criterios de inclusión fueron: pacientes que fueron operados mediante el tratamiento de por lo menos un implante dental en la Clínica de Postgrado de Periodoncia de la Facultad de Odontología UNMSM, pacientes que aceptaron enrolarse de forma voluntario mediante la firma del consentimiento informado entre 18 y 70 ańos. Los criterios de exclusión fueron: diagnóstico de enfermedad periodontal crónica no tratada, diagnóstico de enfermedad sistémica, tratamiento de ortodoncia previa, en gestación o menopausia, reborde menor a $3 \mathrm{~mm}$ en ancho evidenciado en los cortes transaxiales de la tomografía computarizada cone beam (CBCT), reborde menor a $10 \mathrm{~mm}$ en altura evidenciado en los cortes transaxiales de la CBCT, tratamiento de implante previo o regeneración ósea previa.

Se realizó la medición previa del reborde alveolar en un corte transaxial en la CBCT de cada zona del maxilar y mandíbula del paciente. En ese corte se tomó un área con una simulación de un implante 4 x $10 \mathrm{~mm}$ desde el banco de imágenes del programa REALSCAN 2.0 versión 1.0.4.7, midiendo desde el borde superior del reborde alveolar mediante la prolongación de la proyección de la marca radiopaca correspondiente a la guía quirúrgica tomográfica. Las CBCT fueron obtenidas del tomógrafo Point Nix 3D Combi 500s POINTNIX Seúl, Corea. En el área seleccionada se realizó la medición de unidades Hounsfield equivalentes siguiendo el método del fabricante, de acuerdo al siguiente protocolo desarrollado.

En los pacientes seleccionados se procedió a realizar la cirugía de implantes dentales y mediante un colgajo a espesor completo se mostró el reborde alveolar para la colocación de implantes dentales y como acción inicial, se colocó la guía quirúrgica. Se tomaron muestras de 2 
$\mathrm{mm}$ de diámetro y $10 \mathrm{~mm}$ de largo mediante la perforación a través de la guía quirúrgica con una trefina de forma perpendicular al reborde según la orientación de la guía quirúrgica hasta perforar por completo la cortical ósea (Meisinger® $2 \mathrm{~mm} \times 10 \mathrm{~mm}$ ) estandarizada a 800 RPM con un motor ASEPTICO® 7000 y un contrángulo 20:1 DRILLER囚. La muestra obtenida se colocó inmediatamente en un envase estéril conteniendo formol al $10 \%$ para ser etiquetada y enviada al laboratorio de histología de la Facultad de Odontología de la Universidad Nacional Mayor de San Marcos, una vez concluida la cirugía, y preparadas para tinción hematoxilina eosina.

La evaluación histomorfométrica de la muestra se realizó por medio del microscopio óptico de luz, Olympus Bx51 (Tokio, Japón). Se capturaron imágenes con una magnificación de 4x a 40x mediante una cámara digital asociada al microscopio, además se utilizó la técnica de luz polarizada para evaluar la refringencia de las estructuras. La cuantificación de las muestras se realizó con un programa de dominio público (NIH Image, US National Institute of Health).

Los parámetros evaluados en el hueso cortical con una magnificación de 40x y 100x fueron: (1) medición espesor hueso cortical en micras, (2) número de osteocitos en un campo de $100 \times 100$ micras (3) número de sistemas de Havers(osteonas) en un campo de 100 x 100 micras. Los test estadísticos usados fueron medidas de tendencia central y test de correlación de Pearson con el programa STATA versión 14

\section{Resultados}

En la tabla 1 se observa las características de la calidad ósea según sexo. El promedio de las unidades Hounsfield fue de $593 \pm 121,10$ y $570 \pm 127,35$ en el sexo masculino y femenino respectivamente. El número de osteones en promedio fue de $16,5 \pm 2,71$ y $12 \pm 4,68$ en el sexo masculino y femenino respectivamente. El número de osteocitos en promedio fue de $179 \pm 39,99$ sexo masculino y $124 \pm 50,55$ en el femenino (Figuras 1 y 2). El espesor cortical en el sexo masculino fue $33 \pm 50,55$ micras y $31 \pm 8,91$ en el femenino.

En la tabla 2 se observa las características de calidad ósea según regiones del maxilar y la mandíbula. El promedio de unidades Hounsfield fue $557 \pm 232$ en la región premolar superior, $460,67 \pm 1, .01$ en la región molar superior, 593,1 $\pm 59,95$ en la región premolar inferior y $661,7 \pm 120,24$ en la región molar inferior. El número de osteonas fue $9,67 \pm 4,04,11 \pm 4,10,13,7 \pm 3,30$ y $18 \pm 2,83$ en la región premolar superior, región molar superior, región premolar inferior y la región molar inferior, respectivamente. El número de osteocitos fue 117,33 15,14 ,

Tabla 1. Evaluación descriptiva de las unidades Hounsfield, número de osteones, osteocitos y espesor cortical según sexo

\begin{tabular}{|c|c|c|c|c|c|c|c|}
\hline & Sexo & $\mathrm{n}$ & Media $\pm \mathrm{DE}$ & Mediana & Varianza & Mínimo & Máximo \\
\hline \multirow[t]{2}{*}{ U. Hounsfield } & Masculino & 10 & $596,8 \pm 121,10$ & 593 & 14665,96 & 444 & 810 \\
\hline & Femenino & 19 & $579,73 \pm 127,35$ & 570 & 16218,2 & 300 & 852 \\
\hline \multirow[t]{2}{*}{ Número de osteones } & Masculino & 10 & $16,7 \pm 2,71$ & 16,5 & 7,34 & 12 & 20 \\
\hline & Femenino & 19 & $12,89 \pm 4,68$ & 12 & 21,99 & 5 & 20 \\
\hline \multirow[t]{2}{*}{ Número de osteocitos } & Masculino & 10 & $165,5 \pm 39,99$ & 179 & 1599,17 & 119 & 240 \\
\hline & Femenino & 19 & $149,36 \pm 50,55$ & 124 & 2554,80 & 100 & 250 \\
\hline \multirow[t]{2}{*}{ Espesor cortical } & Masculino & 10 & $3,36 \pm 50,55$ & 3,5 & 4,849 & 2,0 & 4,0 \\
\hline & Femenino & 19 & $3,110 \pm 8,91$ & 3,0 & 7,943 & 2,0 & 4,5 \\
\hline
\end{tabular}

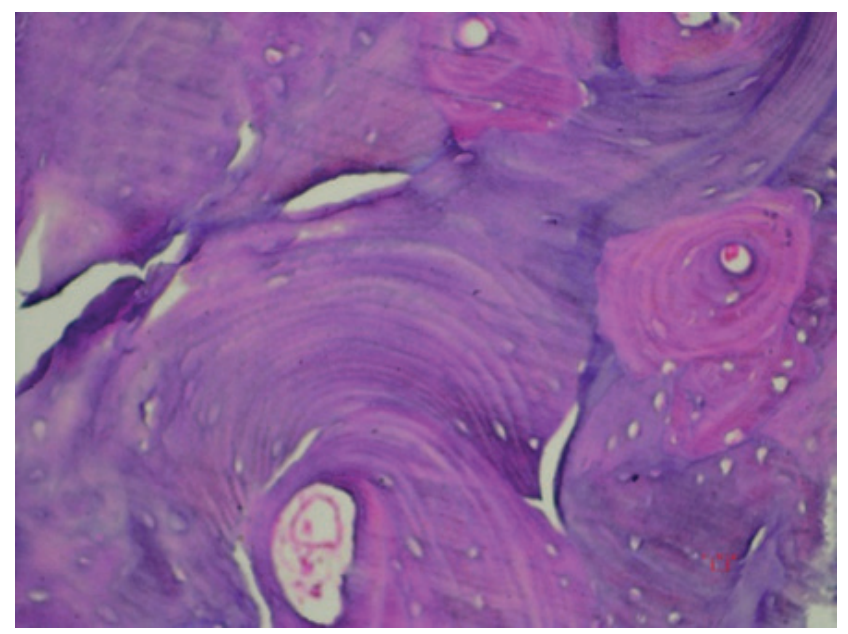

Figura 1. Corte hematoxilina eosina mostrando espesor hueso cortical y la presencia de osteonas 40x

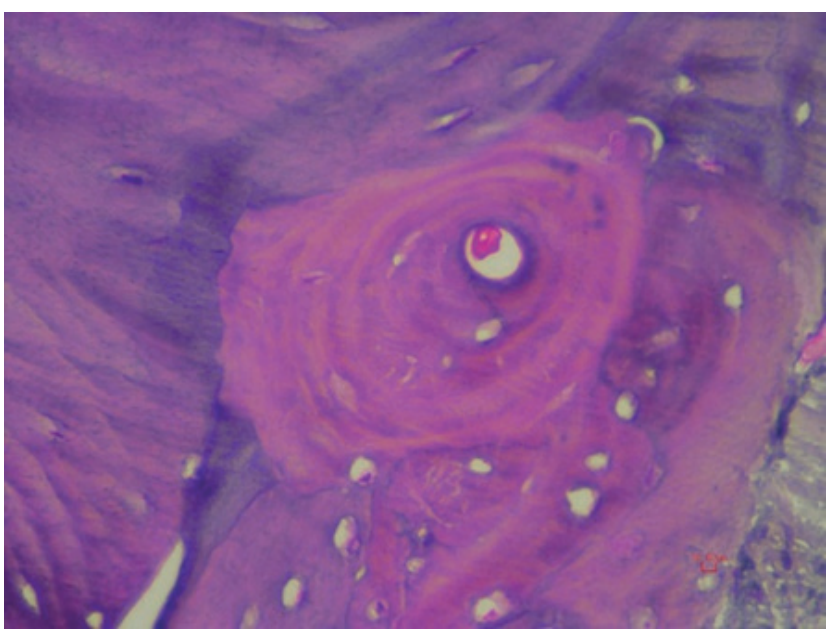

Figura 2. Ampliación de Figura 1. Corte hematoxilina eosina mostrando una osteona y sus capas concéntricas $100 x$ 
Tabla 2. Evaluación descriptiva de las unidades Hounsfield, número de osteones, osteocitos y espesor cortical según región

\begin{tabular}{llcccccc}
\hline & Diente & $\mathbf{n}$ & Media \pm DE & Mediana & Varianza & Mínimo & Máximo \\
\hline U. Hounsfield & Premolar Sup & 3 & $557 \pm 232$ & 620 & 53827 & 300 & 751 \\
& Molar Sup & 6 & $460,67 \pm 15,01$ & 452 & 225,47 & 450 & 481 \\
& Premolar Inf & 10 & $593,1 \pm 59,95$ & 616 & 3594,1 & 444 & 640 \\
& Molar Inf & 10 & $661,7 \pm 120,24$ & 637 & 14458,01 & 543 & 852 \\
Número de osteones & Premolar Sup & 3 & $9,67 \pm 4,04$ & 12 & 16,33 & 5 & 12 \\
& Molar Sup & 6 & $11 \pm 4,10$ & 12 & 16,8 & 6 & 15 \\
Número de osteocitos & Premolar Inf & 10 & $13.7 \pm 3,30$ & 15 & 10,9 & 10 & 17 \\
& Molar Inf & 10 & $18 \pm 2,83$ & 20 & 8 & 14 & 20 \\
& Molar Sup & 6 & $117,83 \pm 4,54$ & 119 & 20,57 & 109 & 122 \\
Espesor cortical & Premolar Inf & 10 & $134,6 \pm 33,44$ & 128 & 1118,27 & 100 & 180 \\
& Molar Inf & 10 & $208,8 \pm 25,40$ & 200 & 645,29 & 179 & 250 \\
& Premolar Sup & 3 & $2,53 \pm 4,62$ & 28 & 2,13 & 2,0 & 2,8 \\
& Molar Sup & 6 & $2,2 \pm 3,098$ & 20 & 0,996 & 2,0 & 2,6 \\
& Premolar Inf & 10 & $3,15 \pm 5,80$ & 30 & 3,36 & 2,0 & 4.0 \\
\hline
\end{tabular}

Tabla 3. Relación de la histomorfometría del hueso cortical con las unidades Hounsfield equivalente en tomografía computarizadas cone beam

\begin{tabular}{lcc}
\hline & $\begin{array}{c}\text { Unidades Hounsfeld } \\
(\mathbf{r})\end{array}$ & $\mathbf{p}$ \\
\hline Espesor cortical & 0,452 & 0,01 \\
Número de osteones & 0,139 & 0,47 \\
Número de osteocitos & 0,339 & 0,07 \\
\hline
\end{tabular}

$117,83 \pm 4,54,134,6 \pm 33,44$ y $208,8 \pm 25,40$ en la región premolar superior, región molar superior, región premolar inferior y la región molar inferior, respectivamente. Y en relación al espesor cortical en $\mathrm{mm} 2,53 \pm 4,61,2,2$ $\pm 3,09,3,15 \pm 5,79$ y $4,04 \pm 2,84$ en la región premolar superior, región molar superior, región premolar inferior y la región molar inferior, respectivamente.

En la Tabla 3 según el valor de $r$ se observa una correlación positiva moderada estadísticamente significativa entre el espesor del hueso cortical y las unidades Hounsfield $(\mathrm{p}<0,05)$. No se observa una relación positiva entre el número de osteones y número de osteocitos y las unidades Hounsfield. ( $p>0,05)$

\section{Discusión}

Con respecto a la calidad ósea se han realizado diversos estudios comparando de forma cuantitativa o cualitativa usando diversos indicadores: el índice de Leckholm y Zarb ${ }^{7,8}$, tomografías con beam ${ }^{9,10}$, radiografías panorámicas ${ }^{11}$, microCT ${ }^{12,13}$, densidad mineral ósea ${ }^{14}$, valores torque de inserción (TVI, por sus siglas en inglés) ${ }^{10}$, muestras tomadas en cadáveres ${ }^{15}$, valores de PERIOTEST® ${ }^{9}$ y coeficiente de estabilidad del implante (ISQ) ${ }^{9,12}$ y elementos finitos ${ }^{18}$.
Leckholm y Zarb propusieron una clasificación del hueso cortical y medular que podría relacionarse con las diferentes zonas del maxilar y la mandíbula en función a un aidferente calidad ósea. En el presente estudio de forma preliminar se observa diferencias en los valores de osteocitos, osteonas, espesor óseo cortical y unidades Hounsfield en las zonas del maxilar estudiadas.

El espesor del hueso cortical fue estudiado por Katranji ${ }^{15}$ hallando los siguientes resultados en zona molar $(\mathrm{M})$, premolar (PM) y anterior (A). Por vestibular, en edéntulos la tabla cortical en el maxilar fue de 1,69 $\mathrm{mm}(\mathrm{M}), 1,43 \mathrm{~mm}$ (PM), y 1,04 mm (A). En el presente estudio, en relación al espesor cortical, los valores en $\mathrm{mm}$ fueron $2,53 \pm 4,61$, $2,2 \pm 3,09,3,15 \pm 0.79$ y $4,04 \pm 2,84$ en la región premolar superior, región molar superior, región premolar inferior y la región molar inferior, respectivamente. Los valores en el estudio de Katranji fueron evaluados en tres regiones: molar, premolar y anterior, en este estudio se ha proyectado a realizar en seis regiones: anterior superior, premolar superior, molar superior, anterior inferior, premolar inferior y molar inferior. Se presentan los resultados preliminares de cuatro regiones obteniendo valores mayores que el promedio en la literatura debido quizá a que no se ha considerado al momento de este estudio las dos regiones anteriores superior e inferior que suelen presentar menores espesores ${ }^{15,16}$. 
Gonzales-García ${ }^{17}$ analizó 39 biopsias de 31 pacientes con cortes tomográficos en microtomografía y los relacionó con biopsias del hueso maxilar hallando una correlación positiva entre ambos análisis concluyendo que la evaluación preoperatoria en tomografías es un método confiable para predecir la densidad del hueso de los pacientes sometidos a implantes. Al momento actual del presente estudio se observa una correlación lineal positiva moderada entre las unidades Hounsfield de la tomografía cone beam y el espesor óseo cortical $r=$ $0,452(\mathrm{p}<0,01)$.

Riberao Rotta et al ${ }^{12}$ tomaron radiografías periapicales y panorámicas de 32 pacientes edéntulos parciales. Tres cirujanos clasificaron la calidad ósea en los sitios de implantes utilizando dos métodos diferentes: evaluaciones en radiografías periapicales y panorámicas $(R P)$ y de acuerdo con la clasificación propuesta por Lekholm y Zarb. Durante la inserción del implante, se tomaron biopsias óseas y se midieron parámetros tridimensionales mediante tomografía microcomputarizada (Micro-CT -por sus siglas en inglés). En este trabajo, hallaron que los tipos de hueso 2 y 3 fueron las clasificaciones más prevalentes según las RP $(54,3 \%)$ e índice de Lekholm y Zarb $(58,7 \%)$.

En el presente estudio, unidades Hounsfield fue $557 \pm 232$ en la región premolar superior, 460,67 $\pm 15,01$ en la región molar superior, 593,1 $\pm 59,95$ en la región premolar inferior y $661,7 \pm 120,24$ en la región molar inferior, eso equivaldría a un tipo D3 según los estudios de Misch ${ }^{6}$ en todas las regiones del presente estudio y a un hueso tipo II según la clasificación de Lekholm y Zarb ${ }^{3}$.

En conclusión, la tomografía computarizada cone beam tiene una correlación con el espesor óseo cortical sin tomar en cuenta las regiones sino los valores globales. Los resultados presentados son preliminares.

\section{Referencias bibliográficas}

1. Lindhe J, Karring T, Lang N. Clinical Periodontology and Implant Dentistry. 5th Editio. Quintessence; 2013.

2. Oh JS, Kim SG. Clinical study of the relationship between implant stability measurements using Periotest and Osstell mentor and bone quality assessment. Oral Surg. Oral Med. Oral Pathol. Oral Radiol. 2012;113(3):35-40.

3. Lekholm U, Zarb GA. Patient selection and preparation. In: Brånemark P-I, Zarb GA AT (eds)., ed. Tissue Integrated Prostheses: Osseointegration in Clinical Dentistry. Quintessence; 1985:199-209.

4. Cardaropoli G, Araujo M, Lindhe J. Dynamics of bone tissue formation in tooth extraction sites. An experimental study in dogs. J. Clin. Periodontol. 2003;30(9):809-18.
5. Newman M, Takei H, Klokkevold, P. Carranza, F. Carranza's Clinical Periodontology. 12th Ed. St Lois: Elsevier; 2015.

6. Misch CE. Density of Bone Effects on surgical approach and healing,. In: Misch C, ed. Contemporary Implant Dentistry Mosby, Elsevier; 2008:645-667.

7. Aranyarachkul P, Caruso MSJ, Gantes MSB, Schulz MSE. Bone Density Assessments of Dental Implant Sites : Int. J. Oral Maxillofac. Implants 2005.

8. Song Y-D, Jun S-H, Kwon J-J. Correlation between bone quality evaluated by cone-beam computerized tomography and implant primary stability. Int. J. Oral Maxillofac. Implants 2008;24(1):59-64.

9. Silva I, De Freitas D, Ambrosano Gi, Bóscolo F, Almeida S. Bone density: Comparative evaluation of hounsfield units in multislice and cone-beam computed tomography. Braz. Oral Res. 2012;26(6):550-6.

10. Pauwels Ruben, Nackaerts O, Bellaiche N, et al. Variability of dental cone beam CT grey values for density estimations. Br J Radiol. 2013;86:1021.

11. Horner $\mathrm{K} \mathrm{DH}$. The relationship between mandibular bone mineral density and panoramic radiographic measurements. J Dent. 1998;26(4):337-43.

12. Gomes de Oliveira RC, Leles CR, Lindh C, et al. Bone tissue microarchitectural characteristics at dental implant sites. Part 1: Identification of clinical-related parameters. Clin Oral Implants Res. 2012;23(8):981-6.

13. Galagali G, Kalekhan SM, Nidawani P, et al. Analysis of the pattern of the alveolar ridge remodelling following single tooth extraction. Clin. Oral Implants Res. 2017;26(1):874-8.

14. Lindh, C. Obrant, K. Petersson A. Maxillary bone mineral density and its relationship to the bone mineral density of the lumbar spine and hip. Oral Surg Oral Med Oral Pathol Oral Radiol Endod. 2008;98(1):102-9.

15. Katranji A, Misch K, Wang H-L. Cortical Bone Thickness in Dentate and Edentulous Human Cadavers. J Periodontol. 2007;78(5):874-8.

16. Sindeaux R, Figueiredo PT de S, de Melo NS, et al. Fractal dimension and mandibular cortical width in normal and osteoporotic men and women. Maturitas 2014;77(2):142-8.

17. González-García R, Monje F. The reliability of cone-beam computed tomography to assess bone density at dental implant recipient sites: a histomorphometric analysis by micro-CT. Clin Oral Implants Res. 2013;24(8):871-9.

18. Escobar JC, Serna JD, Latorre F, Villarraga JA. Evaluación de la microdeformación según diferentes espesores de la tabla ósea vestibular. Odontol Sanmarquina 2018; 21(3):195-204. 
